\title{
UN ACERCAMIENTO EXPLORATORIO EN TORNO A “UNIVERSIDAD Y SIGLO XXI": ASIGNATURA INSIGNIA PARA LA FORMACIÓN INTEGRAL DE LOS DISCENTES, DESDE LA VISIÓN INSTITUCIONAL Y LA MIRADA ESTUDIANTIL
}

\author{
Francisco Javier Bañuelos González \\ Maestro en Gestión y Políticas de Educación Superior \\ Universidad de Guadalajara-CUCEA-México \\ javierb@cucea.udg.mx \\ Blanca Noemí Silva Gutiérrez \\ Doctora en Ciencias \\ Universidad de Guadalajara-CUCEA-México \\ bsilva@cucea.udg.mx
}

\author{
María Guadalupe Villaseñor Gudiño \\ Doctora en Educación \\ Universidad de Guadalajara-CUCEA-México \\ gvillase@cucea.udg.mx
}

\begin{abstract}
Ada Aranzazú Hernández Cuevas
Doctorante en Cooperación y Bienestar Social

Universidad de Guadalajara-CUCEA-México adaah@cucea.udg.mx
\end{abstract}

\section{Cómo citar este artículo}

Bañuelos, F., Villaseñor, M., Silva y Hernández, A. (2013) Un acercamiento exploratorio en torno a la Universidad y siglo XXI: asignatura insignia para la formación integral de los discentes, desde la visión institucional y la mirada estudiantil. Espiral, Revista de Docencia e Investigación. 3 (1) 33 - 44

\section{Resumen}

El presente artículo es un acercamiento exploratorio en torno a Universidad y Siglo XXI, resultado de la primera etapa de una investigación de trayectoria que se realiza por parte de un grupo de profesores especialistas en la temática, cuyo objetivo fue conocer la percepción de los estudiantes de la asignatura "insignia" denominada Universidad y Siglo XXI, para su formación integral. Las reflexiones alrededor de la nueva asignatura, surgen en esta primera fase como un acercamiento a partir del estudio de percepción aplicado a los discentes que ingresaron con la nueva reforma curricular durante el calendario escolar 2013-A al Centro Universitario de Ciencias Económico Administrativas (CUCEA) de la Universidad de Guadalajara (UDEG).

En la UDEG, se consideró pertinente la incorporación de una asignatura "insignia" que brinde a la comunidad universitaria un estado de pertenencia; una visión crítica ante problemáticas en el ámbito local como global; un pensamiento crítico; el desarrollo de herramientas intelectuales, académicas y de investigación; una planeación y prospección de su vida y profesión; así como la construcción de su identidad universitaria, que permita que logren identificar los valores y símbolos, las aspiraciones y anhelos, las costumbres y tradiciones, las prácticas cotidianas y los compromisos sociales, que desde cada ser diversificado convergen hacia un fin común. Bajo estos ambiciosos propósitos, surge el Curso-Taller de "Universidad y Siglo XXI" en una modalidad presencial enriquecida (con la plataforma Moodle), que no debe concebirse como la panacea, sino como la oportunidad que la co- munidad universitaria había esperado para fungir como catarsis de aquellos recovecos ausentes en su formación universitaria. El camino es complejo y el futuro incierto, pero con una visión amplia y pertinente de lo que se quiere y espera de nuestra universidad y de los universitarios, se llegará a construir lo que la sociedad del siglo XXI necesita.

Palabras clave: "Universidad y Siglo" XXI, asignatura insignia, identidad universitaria, percepción estudiantil y formación integral de los discentes.

\begin{abstract}
The present article, it is an exploratory approximation concerning University and 21st century ensued from the first stage of an investigation of path that is realized on the part of a group of teachers specialists in the subject matter, which aim was to know the perception of the students of the subject "emblem" named University and 21st century, for his integral formation. The reflections about the new subject, arise in this first phase as an approximation from the study of perception applied to the discentes that there deposited with the new reform curricular during the schedule of courses 2013-A to the University Economic Center of Sciences Administrative officers (CUCEA) of the University of Guadalajara (UDEG).

In the UDEG, was considered to be pertinent the incorporation of a subject "emblem" that should offer to the university community a condition of belonging; a critical vision before problematic in the local as global area; a critical thought; the development of intellectual, academic
\end{abstract}


tools and of investigation; a planeación and exploration of his life and profession; as well as the construction of his university identity, which allows that they should manage to identify the values and symbols, the aspirations and longings, the customs and traditions, the daily practices and the social commitments, which from every diversified being converge towards a common end. Under these ambitious intentions, there arises the Course-workshop of "University and 21st century " in a modality presencial enriched (with the platform Moodle), that must not be conceived as the panacea, but as the opportunity for that the university community had waited for fungir as catharsis of those absent turns in his universitary education. The way, it is complex and the uncertain future, but with a wide and pertinent vision of what is wanted and expects from our university and from the university students, it will manage to construct what the company of the 21 st century needs.

Keywords: University and 21st century, subject emblem, university identity, student perception and integral formation of the discentes.

\section{INTRODUCCIÓN}

El presente artículo es un acercamiento exploratorio en torno a Universidad y Siglo XXI, resultado de la primera etapa de una investigación de trayectoria que se realiza en el Centro Universitario de Ciencias Económico - Administrativas (CUCEA), por parte de un grupo de profesores especialistas en el nivel superior; sobre temas de educación, gestión institucional, políticas públicas, y tutorías que además, diseñaron e impartieron el curso-taller de Universidad y Siglo XXI, asignatura que es considerada "insignia" por las autoridades institucionales de la Universidad de Guadalajara (UDEG).

El objetivo primordial de esta primera etapa de la investigación fue conocer durante el desarrollo del curso-taller, en una modalidad presencial enriquecida (con la plataforma Moodle); la percepción estudiantil de los discentes que ingresaron al CUCEA-UDEG, con la nueva reforma curricular, que se dio a partir del calendario escolar 2013-A (05-feb. a 15jun.) sobre la utilidad y aplicación en su formación personal, académica, profesional y social, como parte de su formación integral.
Los últimos diez años del siglo XX, fueron de cambio en la educación superior, pues movilizó a las instituciones que habían permanecido desde hacía tiempo en un profundo letargo. Pese a esto, aún permanecen grandes desafíos no resueltos para las universidades, lo cual requiere de un nuevo paradigma de la educación que dé prioridad a la innovación académica, a nuevas estrategias en la generación y transmisión de conocimientos y a una efectiva y pertinente vinculación de la universidad con su entorno (Luengo, 2006).

Por ello, las Instituciones de Educación Superior (IES) no sólo deben adaptarse al cambio, sino prepararse para formar ciudadanos activos, críticos, propositivos y capaces para responder a las necesidades de la sociedad, buscar la sustentabilidad a través del cuidado del medio ambiente, forjar un proyecto que responda a su realidad socio-profesional y que estén comprometidos e identificados con su institución y su entorno (González, 2001).

La inclusión de "Universidad y Siglo XXI", como insignia para la formación integral en el proceso de enseñanza-aprendizaje (PEA), es importante y clave para todo plan de estudios, ya que toda universidad debe contar con un programa sistematizado de construcción y reforzamiento de la identidad institucional que coadyuve a la formación del sentido de pertenencia de su comunidad universitaria (Valdez, 2013).

De acuerdo con Cabral (2006) La identidad estudiantil es un proceso que en ocasiones, se convierte en un problema de gran preocupación para las instituciones de educación superior, ya que repercute positiva o negativamente en la imagen, el prestigio y la calidad educativa a corto y a largo plazo en la comunidad estudiantil, tanto de estudiantes activos, egresados y en la sociedad.

En la Universidad de Guadalajara (UDEG), dentro de su reforma curricular a los planes de 
estudio, se consideró pertinente la incorporación de una asignatura "insignia" que brinde a la comunidad universitaria un estado de pertenencia, una visión crítica ante problemáticas en los ámbitos local y global, un pensamiento crítico y reflexivo, el desarrollo de herramientas intelectuales, académicas y de investigación, una planeación y prospección de su vida y profesión, así como la construcción de su identidad universitaria. Bajo estos ambiciosos propósitos, surge en el calendario escolar 2013 A, la Unidad de Aprendizaje "Universidad y Siglo XXI"

\section{METODOLOGÍA}

En este trabajo de investigación se llevó a cabo un acercamiento exploratorio, resultado de la primera etapa de una investigación de trayectoria, que se realiza por parte de un grupo de profesores especialistas en la temática, cuyo objetivo fue conocer la percepción de los estudiantes de la asignatura insignia denominada "Universidad y Siglo XXI", para su formación integral.

Las reflexiones alrededor de la nueva asignatura, surgen en esta primera fase como un acercamiento a partir del estudio de percepción aplicado a los discentes que ingresaron con la nueva reforma curricular durante el calendario escolar 2013-A al Centro Universitario de Ciencias Económico Administrativas (CUCEA) de la Universidad de Guadalajara (UDEG).

La exploración en esta investigación, está basada en la subjetividad que es una construcción social que depende de los significados que los sujetos le atribuyan (Gómez et al; 2006) como una representación del abordaje cualitativo, ya que a partir de ella se sustenta, se desarrolla y se hace "ser". Pero con una trascendencia de coherencia, rigurosidad y cientificidad a quien aborda esta realidad social más allá de la objetividad (Rusque, 2010).

En este caso el profesor de Universidad y Siglo XXI, como observador, tiene la capacidad de darle a esta subjetividad, una interpretación

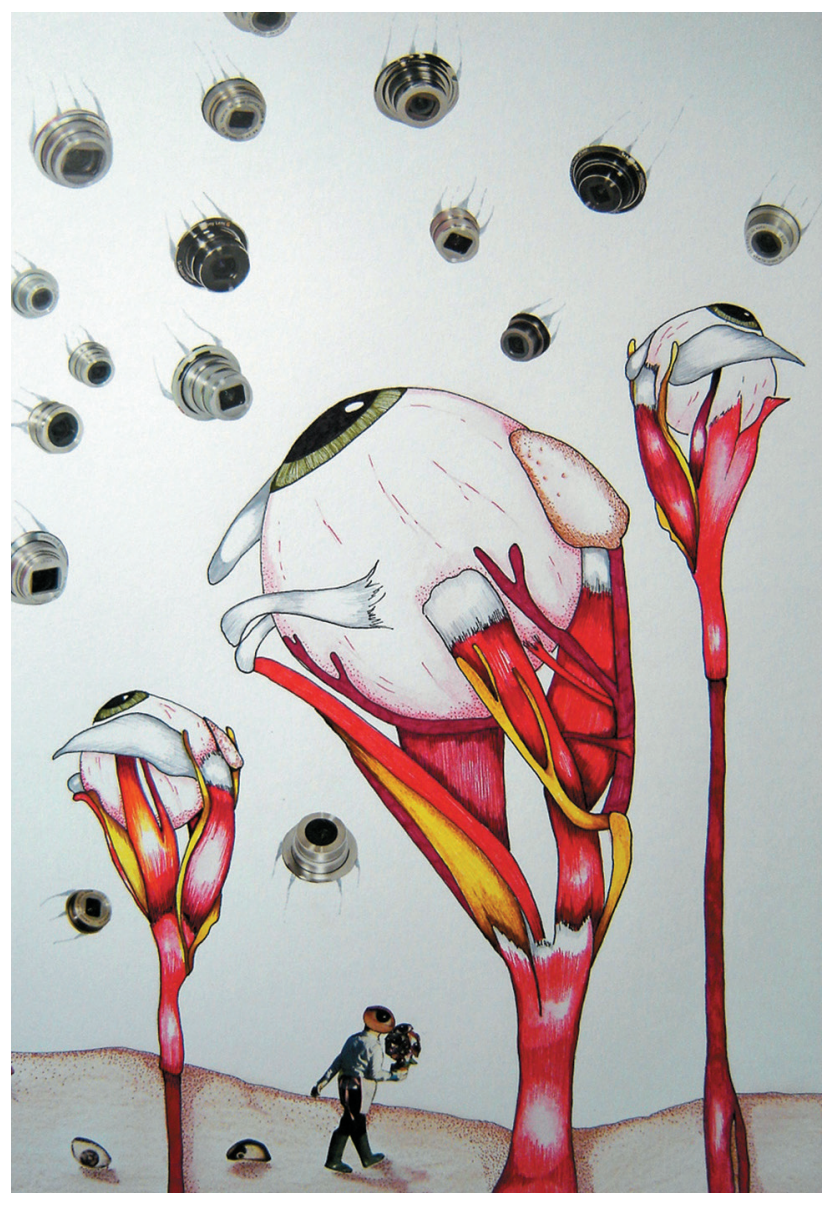

y significatividad a este contexto subjetivo; por ello, se decidió el estudio de percepción de la realidad de los sujetos, ya que es un campo estratégico en el uso de la información por "las organizaciones inteligentes"; las teorías de la organización y de la comunicación han efectuado estudios sobre la percepción donde se menciona que esta permite: la interpretación en relación con el medio ambiente, crean nuevos conocimientos y finalmente procesa la información para aplicarla a cursos de acción. Según el enfoque de estas dos teorías, la percepción es uno de los tres campos estratégicos en el uso de la información pues recibe influencia desde distintos niveles como: el cognoscitivo, el afectivo y el situacional.

Para Choo (1999) las emociones también son importantes en la percepción. En el análisis que hace Weick (1995), a la luz de diversos debates sobre la percepción ésta es un proceso de la organización que posee siete propieda- 


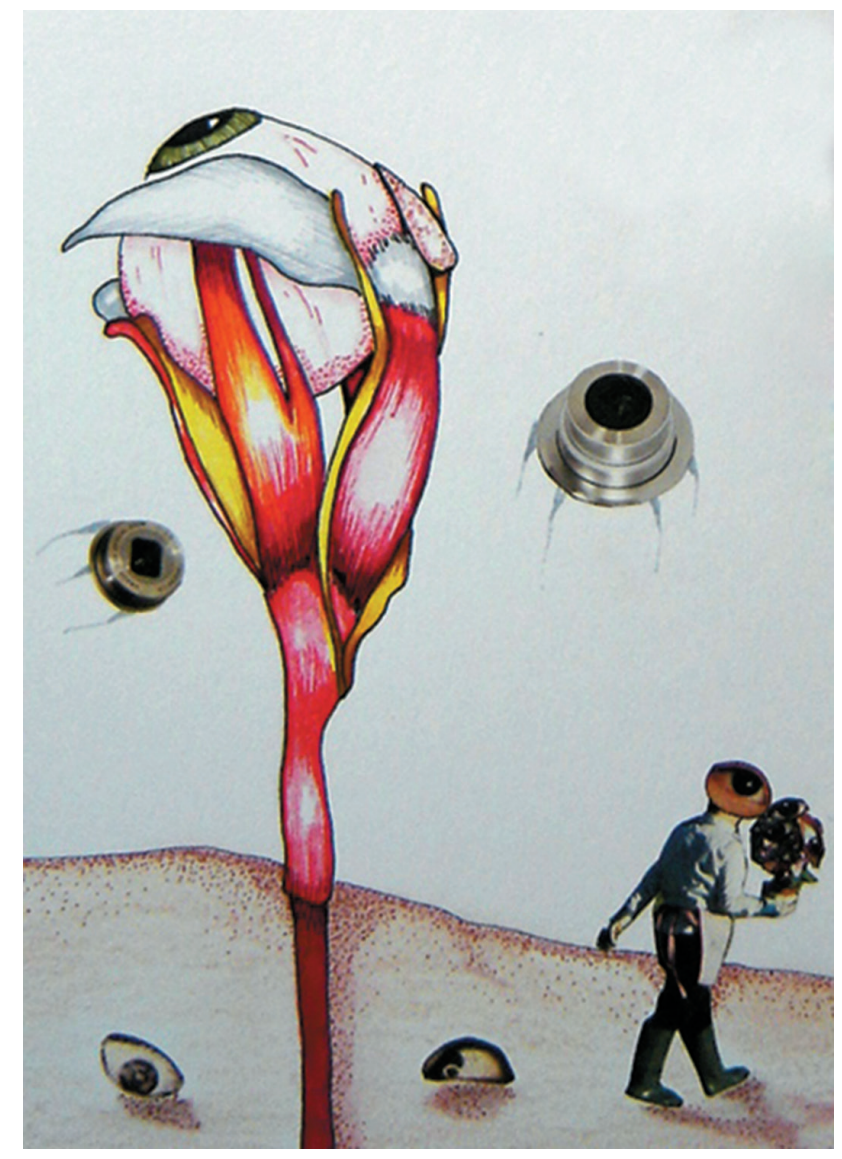

des que la distinguen: 1) se basa en la construcción de la identidad; 2 ) es retrospectivo; 3 ) es representativo del medio ambiente sensible; 4) es social; 5) es constante; 6 ) se concentra en las indicaciones extraídas y 7) es impulsado por la plausibilidad antes que por la exactitud.

A partir de estas condicionantes Milton afirma que las percepciones "no pueden ser falsas ya que la gente simplemente percibe lo que penetra en su conciencia. Lo que luego hagan con esas percepciones a través de la interpretación es un asunto distinto" (Milton 1997: 13). La percepción, entonces, pasa de ser algo que simplemente nos sucede a un proceso en el que intervienen nuestras experiencias y vivencias personales (Milton, 2002).

Por lo anterior, surge la inquietud de conocer desde la subjetividad, la intersubjetividad y la percepción de los discentes de Universidad y Siglo XXI; sobre distintos aspectos, como la pertinencia, la aplicabilidad y trascendencia del curso-taller para su formación integral. Para el logro de este objetivo, desde las bases de la subjetividad, se realizó un estudio de percepción aplicado a 4 cuatro grupos de estudiantes, conformado por 50 sujetos cada uno, que representó el $10 \%$ de los estudiantes de nuevo ingreso $(2,201)$ en el calendario escolar 2013-A, de las licenciaturas de Turismo, Negocios Internacionales, Tecnologías de la Información, Recursos Humanos, Contaduría, Mercadotecnia, Administración Financiera y Sistemas, Administración Gubernamental y Políticas Públicas, Gestión y Economía Ambiental, Economía y Administración.

Este estudio se realizó mediante la aplicación de un instrumento conformado por varias categorías para analizar cuestiones institucionales, de identificaciones de rol universitario, prospectivas de vida y profesionales. Además la observación directa dentro de cada uno de los grupos, permitió el análisis de aspectos referentes con la intencionalidad del curso-taller y cuestionar el cumplimiento del propósito institucional.

\section{Universidad y Siglo XXI: un medio para cons- truir la Identidad Universitaria}

La identidad universitaria es un tema de relevancia para las instituciones de educación superior. El comprender el grado de identidad de los universitarios sirve para generar estrategias que produzcan una mayor identificación del alumnado hacia su universidad, que impulse el crecimiento institucional hacia dentro y fuera de la misma (Cabral et al; 2006).

Las Instituciones de Educación, deben impulsar acciones orientadas a generar y acrecentar el afecto, reconocimiento, orgullo, y gratitud hacia su Alma Mater (Valdez, 2013); esto se logra mediante la construcción de una identidad institucional que sea compartida y adoptada por la comunidad educativa. Dicha construcción se da mediante el proceso de identificación que vive el estudiante universitario den- 
tro de la dinámica de relación que establece con sus nuevos compañeros, los docentes, la misma institución, su medio social (Brubaker \& Cooper, 2001) y la territorialidad, entendida como base al desarrollo del sentido de identidad personal y de grupo; por lo que, tener instalaciones acordes con las necesidades de los estudiantes y espacios donde los estudiantes se sientan parte de ella y tomen la defensa de estos espacios, generarán un sentido de pertenencia más sólido, ya que ellos señalarán su territorio para identificarse con él mismo y los demás (Holohan, 2003).

Según Luque (2003), existen otros factores que integran esta construcción de la identidad como la experiencia escolar pasada, la pertenencia, las relaciones humanas, la percepción de la universidad antes del ingreso, las afinidades con el medio, el método de enseñanza, las reglas y la dificultad de acceso a la institución, son aspectos que intervienen en la construcción de la identidad institucional.

Esta identidad antes construida, está en constante redefinición, al paso de la vida personal y académica, ya que el sujeto tiene la capacidad de construir nuevas relaciones por medio de su subjetividad, que nunca será una unidad terminada ni cerrada. Debido a que la identidad se construye a cada momento en la medida de cada vivencia individual, esta se transforma en sustento o en experiencia pasada, el cúmulo de vivencias académicas que se desarrollan a lo largo de la vida académica, dentro de ambientes institucionales con maestros, estudiantes y la propia familia; orillan a una constante redefinición y reconstrucción por los individuos mismos y las instituciones son las encargadas de definirla (Brubaker \& Cooper, 2001).

La identidad institucional se refiere a formar parte de algo a partir de un modelo, con la conciencia de pertenencia y adoptar características de un grupo, componentes que se refuerzan por el prestigio y la calidad de la universidad, donde los estudiantes tienen un objetivo y una visión que los hace participar e involucrarse con la institución, siendo así personas comprometidas con ésta y con su profesión. Esto se da con base en el interjuego que se crea dentro de la universidad, la cual trata de satisfacer las necesidades de los educandos al crear la imagen del estudiante, haciéndoles sentir orgullo, felicidad y agrado porque las instalaciones de su universidad cuentan con estándares de calidad altos para el desarrollo integral de los estudiantes (Cabral, et al; 2006).

Para Jerkins (1996), la identidad universitaria no es unilateral, se construye en múltiples dimensiones según la práctica social de los individuos que tienen esta práctica social dentro de un ambiente institucional, que es la universidad.

El logro de una identidad implica sentido de pertenencia y afinidad hacia algo, brinda ubicación y confort a todo individuo, es parte de la naturaleza humana el ser o formar parte de algo y más cuando se comparten cuestiones históricas, valores, relaciones afectivas, tradiciones y simpatía; estos aspectos canalizados de manera asertiva, provocan un compromiso de los individuos con su sociedad.

De acuerdo a lo anterior la identidad universitaria se entiende como:

"(...) el conjunto de repertorios culturales compartidos por una comunidad universitaria a partir de los cuales se definen a sí mismos, orientan sus acciones y otorgan sentido a sus prácticas cotidianas (...)" o como "(...) el conjunto de elementos, caracteres y circunstancias propias de una universidad, que la hacen distinta, diferente a las demás universidades, y que se manifiesta a través de símbolos que representan los valores que aspiramos hacer nuestros como universitarios" (Valdez, 2013)

Para esta investigación la identidad universitaria es el cúmulo de referentes externos e internos que el individuo apropia dentro de su 
práctica social; a partir de la subjetividad y de las interacciones y relaciones con su medio, llega a la construcción de una identificación. Ésta no resulta del simple hecho de ser miembro de una comunidad o pertenecer a un grupo social. Lograr desarrollar y construir una identidad universitaria es un proceso complejo de construcción de significados, donde se logre identificar los valores y símbolos, las aspiraciones y anhelos, las relaciones e interacciones, las costumbres y tradiciones, las prácticas cotidianas y los compromisos sociales, que desde cada ser diversificado convergen hacia un fin común.

La Universidad de Guadalajara es una institución bicentenaria que ha cumplido un papel vital y estratégico para el desarrollo educativo y social. Por ello, la implementación de Universidad y Siglo XXI, en los planes de estudio de la UDEG, era necesario, para otorgar una formación integral; ya que de sus aulas han egresado generaciones de profesionales, que día a día se integran al sector productivo, sirven a la sociedad y en varios casos generan conocimiento y desarrollo tecnológico. La aportación al desarrollo económico y social que ha desencadenado la UDEG es innegable; sin embargo, resulta exigente fomentar, fortalecer y potencializar la identidad universitaria, como política institucional que haga frente a los nuevos retos y desafíos del mundo globalizante y cambiante (Valdez, 2013).

La tarea de las Instituciones de Educación Superior (IES) es formar profesionales capaces de responder a las necesidades de su época y a vislumbrar y considerar las futuras; sin embargo, la incorporación de una asignatura "insignia" dentro del proceso de formación no sólo depende de los conocimientos y habilidades que desarrolló el estudiante en el contexto universitario, sino también de los valores e intereses particulares que regulan su actuar.

El curso de "Universidad y Siglo XXI", está considerado para la Universidad de Guadalajara (UDEG) como una asignatura insignia que busca desarrollar una formación integral en los jóvenes universitarios, herramientas y habilidades para el mundo competitivo y que en el desarrollo de esta unidad aprendizaje, adquieran e identifiquen su identidad universitaria, que comprendan cuál es su rol como universitarios en el siglo XXI y analicen su compromiso con la sociedad, como sujetos cognoscentes y practicantes de una profesión para el mejoramiento y bienestar social.

En síntesis, mantener, construir y apropiarse de una identidad universitaria, es como afirma Solís Gadea (2013), "la herencia sin testamento", que está allí para ser recuperada, guiarnos y llevarnos hacia el futuro.

\section{Visión Institucional sobre la asignatura Uni- versidad y Siglo XXI, en la UDEG}

La visión institucional respecto a esta asignatura que se ha incorporado recientemente, a partir del calendario escolar 2013-A en algunos campus ${ }^{1}$ temáticos y regionales de la Universidad de Guadalajara, es que esta asignatura "insignia" como la han denominado las autoridades institucionales, les brindará a los estudiantes de primer ingreso a su formación universitaria, una integralidad en el ámbito académico, profesional, social y personal; aseveración que es aventurado afirmarse y parte de lo cual ocupa esta investigación.

El calendario escolar 2013 A, llama a profundizar en lo alcanzado y a la búsqueda de posicionar a la Universidad de Guadalajara (UDEG), entre las 10 mejores universidades de Iberoamérica; llegar a esa posición será el resultado de los esfuerzos, los logros de una lucha conjunta y el reforzamiento de nuestra identidad (Solís, 2013). Es por ello, que el peso que recae sobre "Universidad y Siglo XXI", obliga a

1 Los campus que han incorporado la asignatura de universidad y siglo XXI a su malla curricular como básica común obligatoria para las carreras a nivel pregrado que ofrecen son: el Centro Universitario de Ciencias Económico Administrativas (CUCEA), el Centro Universitario de Tonalá (CuTonalá), Centro Universitario de los Altos (CuAltos), Centro Universitario de la Ciénega (CuCiénega), Centro Universitario de la Costa(CuCosta), Centro Universitario de la Costa Sur (CuCostaSur), Centro Universitario del Sur (CuSur), Centro Universitario de los Valles (CuValles), Centro Universitario del Norte (CuNorte) y Centro Universitario de los Lagos (CuLagos). 
los profesores y a los estudiantes y las autoridades encargadas de encauzarla efectivamente, a redoblar esfuerzos y poner especial atención en su ejecución.

El curso de Universidad y Siglo XXI, es de orden práctico, en la modalidad de curso-taller; que garantiza que los educandos, adquieran las habilidades, herramientas y aspectos formativos necesarios para integrase plenamente en el contexto universitario y puedan desarrollarse con éxito hasta la culminación de su formación universitaria. El mismo está constituido por 5 ejes formativos y un factor inherente que de manera paralela, está íntimamente ligado al curso en el ámbito de la evaluación y es la tutoría.

Los ejes formativos que constituyen el programa de Universidad y Siglo XXI, es el de "Universidad presente, pasado y futuro"; en este eje el objetivo es que los estudiantes adquieran una visión amplia sobre la transformación que se han suscitado en torno a las universidades como instituciones en pro del desarrollo y bienestar social; además que identifiquen y construyan su propia identidad universitaria, comprendan cuál es su rol como universitarios y finalmente su compromiso social, para la construcción de una sociedad mejor.

El eje de "autogestión del conocimiento y habilidades de aprendizaje", busca promover y estimular habilidades y destrezas en aspectos de investigación, pensamiento reflexivo y crítico, construcción de significados y saberes, fomentar el interés y la aplicabilidad de la lectura, la autodisciplina y el mejoramiento eficiente del aprendizaje a través de la identificación propia de sus estilos de aprendizaje.

Los ejes sobre "Sociedad global: problemas y soluciones transversales" y "México y Jalisco: necesidades sociales y alternativas de desarroIlo", de manera transversal orillan a que los estudiantes logren identificar la relación que existe entre el plano local y el global con la intención que se comprenda la influencia (positiva o ne- gativa) que tiene el sistema internacional en el escenario local, para que, a partir de ello puedan conocer la situación socioeconómica de México y en particular de Jalisco mediante el análisis teórico y metodológico, con el fin de reflexionar sobre la realidad objetiva de la cual forma parte el estudiante, como ciudadano y profesionista en la sociedad actual.

Al país lo aquejan enormes problemáticas y necesidades, que en cierto sentido se convierten en áreas de oportunidad para crecer como institución y vigorizar el liderazgo social que tiene la UDEG. En este sentido, se debe trabajar para aprovechar estas oportunidades, alinear los recursos y las capacidades con las que cuenta la UDEG y simplemente "dejar huella en la sensibilidad de la época" (Solís, 2013).

El eje sobre "vida, profesión y vocación" permite vislumbrar cuáles son los retos actuales y las perspectivas de desarrollo profesional particularizado, para desarrollar su proyecto de vida e identificar el sentido de vocación en su profesión, en torno a las competencias educativas y profesionales de cada carrera. Este eje ha estado sujeto a polémica, debido a que es considerado preparatorio o de bachillerato; sin embargo, las experiencias y percepciones de los estudiantes que la han cursado, difieren de las anteriores.

\section{La mirada de los estudiantes del CUCEA res- pecto al curso Universidad y Siglo XXI}

La mirada estudiantil en torno a Universidad y Siglo XXI, estuvo en su primer lanzamiento envuelto por expectativas, críticas, confusiones, desánimos, apatía y en muchos de los casos aprovechamiento; estas cuestiones fueron emergentes debido a que era la primera vez que reflexionaban y trabajaban sobre estos tópicos, aunque no fueran desconocidos, aspecto que resultó difícil responder y realizar a los cuestionamientos y prácticas. Las dinámicas y actividades realizadas en este curso-taller, como parte de la estrategia metodológica, se 
convirtieron en un buen pretexto que favoreció el acceso a la información relevante e imposible de extraer por otros medios y, además, estimuló y desarrolló la adquisición de habilidades cognitivas, de análisis y de aprendizaje; plantearon un proyecto de vida sustentable, se identificaron con su institución y comprendieron su rol como universitarios del Siglo XXI. Como es propio de los fenómenos complejos, este estudio en su primera etapa originó más interrogantes que respuestas.

Los nuevos estudiantes en Universidad y Siglo XXI, perciben a la universidad como una institución conformada por estudiantes y maestros orientados a un fin común, el de la formación de jóvenes tanto profesional como éticamente durante su estancia, además es un lugar donde el pensamiento se vuelve crítico y permite decidir consciente y libremente sobre cómo actuar en la sociedad.

Cuando se les cuestionó: ¿Cuál es la misión de la universidad en el siglo XXI, desde su percepción? El común fue que la misión de la Universidad en el siglo XXI es formar alumnos altamente capacitados, tanto en el aspecto profesional como en el de investigación, con calidad humana y académica, así como difundir el desarrollo y conocimiento para el logro de una sociedad justa, solidaria y productiva.

De acuerdo a lo anterior; para ellos, la Universidad del Siglo XXI debe ser equitativa, incluyente, con un alto nivel de calidad en educación, deben ser autónomas y muy dinámicas, donde se aplique el sentido de la innovación; ya que manifiestan que este factor (innovación) representa cambios fundamentales en la institución y descubrimientos científicos y tecnológicos. Por su parte, la función de la Universidad del Siglo XXI, la conciben como la de crear y desarrollar los conocimientos para formar ciudadanos comprometidos y capaces, que apliquen razonadamente técnicas y métodos útiles a la sociedad presente y futura.
Una pregunta donde la respuesta se abría en tres vertientes es sobre cuál es la responsabilidad en la sociedad de las Universidades como institución, del gobierno y de los propios estudiantes; un interrogante que deja mucho a la reflexión, que reafirma los retos pendientes y destapa las necesidades urgentes para una "sociedad mejor" que todos buscan, anhelan. ¿Pero quién realmente trabaja para alcanzarla? Bajo esta inquisitiva, estas fueron las percepciones:

La universidad tiene como responsabilidad la formación de ciudadanos capaces de pensamiento y de acción inteligente, decididos y realizadores, pero no impulsivos; que permee en el colectivo estudiantil la conciencia y el gusto por la contribución moral, intelectual, técnica y de recurso humano, para afrontar las necesidades y promover alternativas de desarrollo social.

La responsabilidad social del gobierno es invertir en la educación, ciencia y tecnología; así como dar apoyo a estudiantes destacados en la participación de proyectos inteligentes y la implementación de políticas que permitan un bienestar en la sociedad.

Los estudiantes tienen como responsabilidad ser autogestivos, autónomos, tener iniciativa por consultar e investigar, que sean innovadores, emprendedores, deben ser lectores por naturaleza, responsables, críticos, competentes, constructores de su propio aprendizaje y generadores de soluciones sociales.

Una de las partes más importantes es donde identificaron su rol, su compromiso y su identidad como universitarios en el siglo XXI, esta parte reafirma y cumple la intencionalidad de la incorporación de la asignatura en la formación universitaria, porque los estudiantes al finalizar el curso en el ciclo escolar 2013 A, lograron identificar su rol universitario, comprendieron cuál es su compromiso con la sociedad y construyeron su identidad universitaria, que sin duda alguna fortalecerá su desenvolvimien- 
to y desempeño estudiantil, personal, profesional y social.

"Ser universitario en el siglo XXI es tener una amplia visión, así como una mentalidad más abierta, capaces de aprovechar todos los medios para una mejor formación intelectual, afectiva y social, ya que la competencia cada día es mayor (A1C203)"

El universitario en el siglo XXI identifica que tiene como responsabilidad buscar autonomía en sus propios aprendizajes, ser conocedor de problemáticas sociales, donde el principal compromiso es contribuir al cambio, ser capaces de resolver problemas y crear conciencia que forman parte del motor impulsor y son coresponsables del futuro de nuestro país.

"Mi identidad universitaria es sobresalir ante todo, cumplir todas mis metas, compartir mis conocimientos, tener iniciativa por investigar, crear y desarrollar. Ser competente y aprender de todas mis experiencias durante la universidad (A15C201)".

Construir y adoptar una identidad universitaria, tiene para el estudiante un significado especial y relevante, ya que en ella está inmersa la tradición, la costumbre, las vivencias, los deseos, además que esto le permite reconocerse diferente pero "tirando hacia un mismo lado".

\section{CONCLUSIONES}

En la actualidad se habla de una sociedad en crisis de valores de todo tipo; una desvalorización en el respeto, gratitud y reconocimiento por su Universidad, se hace cada vez más presente en los susurros populares de la propia comunidad universitaria, desacreditando la ardua labor y la misión que ésta conlleva; efecto de un desconocimiento universitario, de una falta de identidad universitaria, no comparten un sentimiento de aprecio común por su universidad, donde en el transitar universitario no logran identificar su rol y compromiso como seres universitarios.

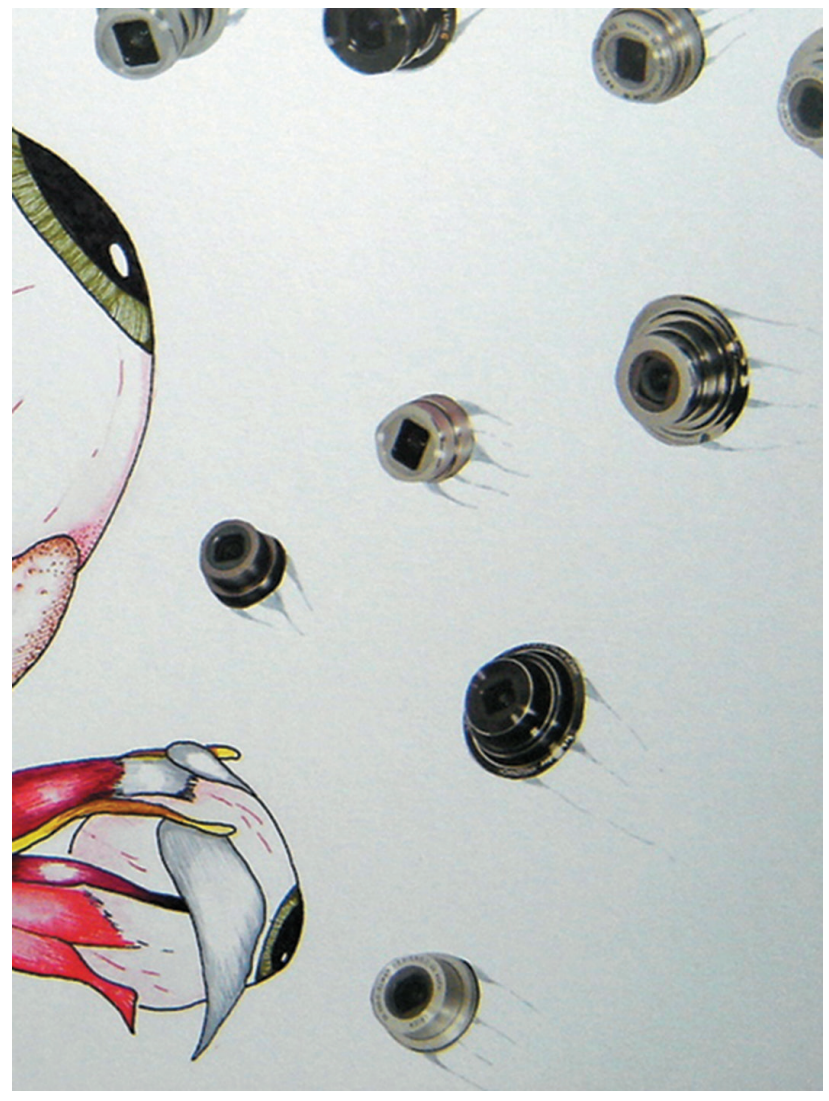

El fin del curso de Universidad y Siglo XXI, no es simplemente implementar en ellos una identidad universitaria, sino la creación de habilidades, destrezas y competencias necesarias para su transitar durante la estadía universitaria y para responder a las necesidades sociales del Siglo XXI. Sin embargo, la construcción de una identidad universitaria en los discentes empata con la función social de las instituciones.

Respecto a la construcción de la identidad en los estudiantes también es necesario hacer algunos replanteamientos. Debe considerarse que ésta no solamente comprende la configuración de los valores, sino las formas y estrategias por medio de las cuales los hacemos parte de los contextos culturales. La visión sobre el entorno y la apreciación que tenemos al respecto son aspectos primordiales para hacer suyos éstos y no otros valores y estrategias. Asimismo, hay que enfatizar la reflexión sobre la dimensión social, y reforzar los estilos de interactuar con el entorno de manera que se llegue a potenciar el compromiso y el cultivo de los valores. El reto es transfor- 


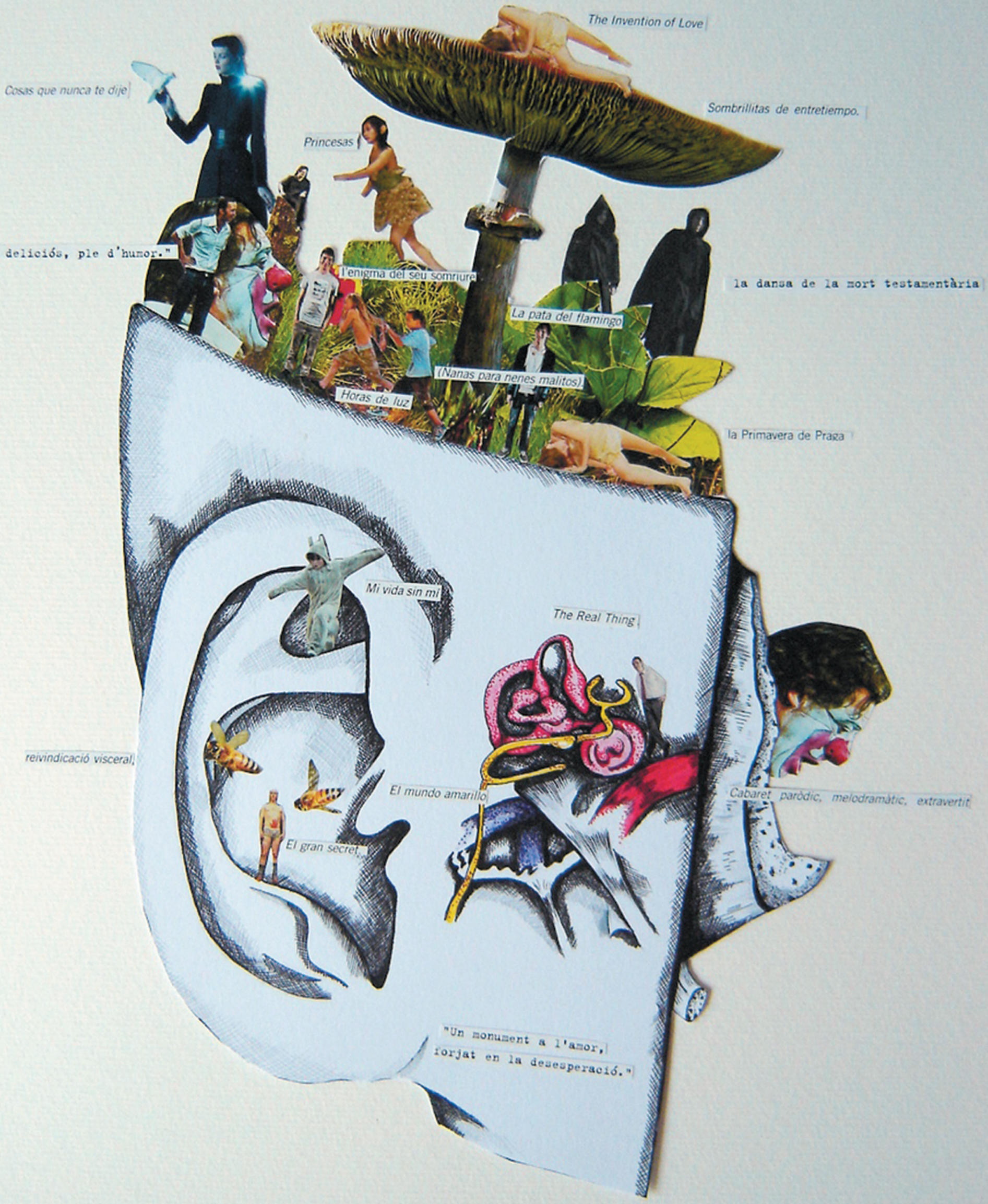


mar la apatía social y política en una educación para la participación ciudadana comprometida.

Con visión crítica se espera que los resultados de incorporar Universidad y Siglo XXI, en la formación universitaria, despierte la inquietud, interés y el compromiso que la comunidad universitaria tiene con la sociedad, que estimule realmente la participación ciudadana comprometida que desde hace algún tiempo permanece dormida. La construcción de una identidad universitaria va más allá de ponerse una camisa con el escudo, de asistir a un partido de fútbol, del simple hecho de mencionar que eres parte "de" o de gritar con énfasis una porra; la identidad universitaria debe ser demostrada, que la sociedad vea esa identificación en la unión de sus miembros, en la solidaridad en los desastres sociales, en actividades de labor social, en promoción del cuidado del ambiente, en el servicio profesional solidario y en un actuar ético y responsable con los demás.

La experiencia del primer grupo de estudiantes $(2,220)$ que ingresaron al CUCEA, en el calendario escolar 2013 -A, estuvo envuelta de expectativas, críticas, sugerencias y recomendaciones, que sería aventurado afirmar que cumplió con las exigencias y la intencionalidad para la que fue creada la asignatura; sin embargo, el esfuerzo y el compromiso por los sujetos directamente involucrados, es incuestionable. Es pertinente mencionar que en el transcurso de la implementación del PEA, surgieron aspectos a mejorar como en todo proceso innovador que recién ha sido implementado.

La información obtenida muestra que las expectativas de los estudiantes, fueron superadas, lograron identificar su compromiso social y su rol como universitarios, construyeron y adoptaron su identidad universitaria; desarrollaron habilidades de autogestión, de aprendizaje, de investigación; analizaron problemáticas sociales, para darle alternativas de solución y desarrollo y finalmente, desarrollaron un proyecto de vida, reafirmaron su vocación, en algunos casos, el eje sobre vida, profesión y vocación, les ayudó a desistir y convencerse que estaban en el camino (profesión) inadecuado, y a identificar y conocer las exigencias y necesidades que implica la práctica de su profesión.

El curso-taller de Universidad y Siglo XXI, en su primer implementación, reafirmó que el contenido que se maneja en esta asignatura, acompañará implícitamente el andar del quehacer como individuo y profesional, la intención de éste radica en la necesidad de que el discente incorpore y desarrolle valores, habilidades, destrezas y aspectos formativos para que posteriormente pueda plasmarlos en todos los matices de sus proyectos de vida personal, académica, profesional y social.

En el contexto globalizado y otras tendencias homogeneizadoras, así como en sociedades manipuladas por la producción y el consumo, el aprender a ser, representa uno de los propósitos no cumplidos por la educación. La flexibilidad,la tolerancia, la apertura, el dinamismo y la dimensión experimental y vivencial, propia de los procesos de la transformación social, pueden encontrar cabida en una asignatura "insignia" como "Universidad y Siglo XXI", que permite y busca potenciar a los sujetos para el desarrollo y la lucha por la igualdad, la equidad, la sustentabilidad, la dignidad humana y el bienestar social.

Universidad y Siglo XXI, no debe concebirse como la panacea que solucionará los tropiezos que se han desencadenado en la UDEG, ni por si sola formará los profesionistas que la sociedad espera; sin embargo, es la oportunidad que la comunidad universitaria había esperado para fungir como catarsis de aquellos recovecos sueltos que hacían falta en su formación universitaria

El camino, es complejo y el futuro incierto, pero con una visión amplia y pertinente de lo que se quiere y espera de nuestra universidad y de los universitarios, se llegará a construir lo que la sociedad del siglo XXI necesita. 


\section{BIBLIOGRAFÍA}

Brubaker, R. \& Cooper, F. (2001). Más allá de la identidad. Apuntes de Investigación del CECYP, Vol. 7, pp. 30-67.

Cabral, M., Villanueva, E., Estrada, G., González, S. R., Juárez, C., Hernández, C.,Flores, M. \& Nácar, V.(2006, 08 de noviembre). Identidad estudiantil universitaria en estudiantes de licenciatura.Revista PsicologiaCientifica.com, 8(16). Disponible en: http://www.psicologiacientifica.com/identidad-estudiates-universitarios

CUCEA (2010). Portal oficial del Centro Universitario de Ciencias Económico-Administrativas (CUCEA). Disponible en http://www.cucea.udg.mx

CUCEA (2013).Programa asignatura Universidad y Siglo XXI. Disponible en: http://www.cucea.udg. $\mathrm{mx} / \mathrm{q}=$ bitacora/consulta-en-I-nea-de-los-nuevosprogramas-de-asignatura

Choo, Chun Wei. (1999). The Knowing organization: How organizations use information to construct meaning, create knowledge and make decisions International. Journal of Informations Management, 16(5). Oxford: Oxford University Press. Disponible en http://choo.fis.utoronto.ca/FIS/respub/ IJM1996.pdf

Delors, J. (1996). La Educación encierra un tesoro, Informe a la UNESCO de la Comisión Internacional sobre la educación para el siglo XXI. Madrid: Santillana, Ediciones UNESCO.

Gómez, J., Latorre, A., Sánchez, M. y Flecha, R. (2006). Metodología Comunicativa Crítica. Barcelona: El Roure Ciencia.

González, P. (2001). La Universidad necesaria en el siglo XXI. México: Era

Holahan, C. (2003). Psicología ambiental: un enfoque general. México: Limusa Noriega Editores.

Jenkins, R. (1996). Social Identity Theorising social identity. Inglaterra: Routledge.

Kepowics, B. (2003). Valores en los estudiantes universitarios. En: Revista Reencuentro no. 38. Recuperado de http://bibliotecadigital.conevyt.org.mx/servicios/ hemeroteca/ reencuentro/no38/index.html

Luengo, E. (2006). Posibilidades de una nueva institucionalidad social: contribución desde la educación y el conocimiento. En Nuevos retos y derroteros para la educación superior en el siglo XXI. México: Universidad de Guadalajara.
Luque, M. (2003). Educación, multiculturalismo e identidad. España: Universidad de Lleida.

Milton, K. (1997). Estudio metodológico basado en el enfoque de percepciones. Disponible en: http://www. uam-antropologia.info/alteridades/alt8-4-vargas. pdf

Milton, K. (2002). Loving Nature. Towards an Ecology of Emotion. London: Routledge. Disponible en http://www.deepdyve.com/lp/sage/book-reviewloving-nature-towards-an-ecology-of-emotionBLk3RAQre2.

Morín, E. (1999). Los siete saberes necesarios de la educación del futuro. Paris, Francia: UNESCO. Disponible en http://www.bibliotecasvirtuales.com/biblioteca/articulos/ los7saberes/index.asp.

Morse, J. Editora (2003). Asuntos críticos en los métodos de investigación cualitativa. Antioquia: Universidad de Antioquia.

Piñero, M. (2012). Implicaciones de la subjetividad e intersubjetividad en el proceder de la investigación cualitativa. Recuperado de http://boards5.melodysoft.com/ ForoDoctoradoUNY2012-1/re-introduccion-a-la-introduccion-92.html

Rusque, A. (2010). De la Diversidad a la Unidad en la Investigación Cualitativa. Caracas: Vadell Hnos. Editores, C.A.

Solís Gadea, H.R. (2013). “La universidad de Guadalajara del Siglo XXI"En la Gaceta UDEG. Año 11, edición 732. 04 de febrero de 2013. Disponible en: http:// gaceta. udg.mx/G_nota1.php?id=13331

Universidad de Guadalajara. "Informe de actividades 2012-2013 del rector del CUCEA, Mtro. Itzcóatl Tonatiuh Bravo Padilla". Disponible en: www.udg.mx

Valdez, A. (2013) a. "Identidad universitaria" En la Gaceta UDEG. Año 11, edición 734. 18 de febrero de 2013. Disponible en: http://gaceta.udg.mx/G_nota1. php?id=13427

Valdez, A (2013) b. ¿Qué Significa ser UDEG? En la Gaceta UDEG. Año 12, edición 754. 22 de julio de 2013. Disponible en: http://gaceta.udg.mx/G_nota1. php?id=14357

Weick, K. (1995). Sensemaking in organizations. Sage. Thounsand Oaks. California. Disponible en http:// www.utwente.nl/cw/theorieenoverzicht/Theory $\% 20$ clusters/ Organizational\%20Communication/Sensemaking.doc/. 